\title{
Thermophoretic force and velocity of nanoparticles in the free molecule regime
}

\author{
Zhigang Li and Hai Wang \\ Department of Mechanical Engineering, University of Delaware, Newark, Delaware 19716, USA
}

(Received 25 February 2004; published 31 August 2004)

\begin{abstract}
We extend our previous gas-kinetic theory analysis of drag force in a uniform temperature field [Li and Wang, Phys. Rev. E. 68, 061206 (2003); 68, 061207 (2003)] to particle transport in fluids with nonuniform temperature. Formulations for drag and thermophoretic forces are proposed for nanoparticle transport in lowdensity gases. We specifically consider the influence of nonrigid body collision due to van der Waals or other forces between the particle and gas molecules and find that these forces play a notable role for particles a few nanometers in size. It is shown that the present formulations can be easily reduced to the classical result of Waldmann [Z. Naturforsch. A 14a, 589 (1959)] by assuming rigid body collision. From the force formulations we also obtain the equation governing the thermophoretic velocity. This velocity is found to be highly sensitive to the potential energy of interactions between gas molecules and particle, and as such Waldmann's thermophoretic velocity is not expected to be accurate for nanosized particles.
\end{abstract}

DOI: 10.1103/PhysRevE.70.021205 PACS number(s): 47.45.Dt, 05.20.Dd, 05.60.Cd, 65.80.+n

\section{INTRODUCTION}

In recent studies [1,2] we obtained the mathematical formulations for the drag force, diffusion coefficient, and electric mobility of small particles in a fluid with a uniform temperature and a small particle Reynolds number $(\operatorname{Re}<1)$. In the free molecule regime our analysis showed a notable influence of the van der Waals and other forces between the particle and fluid molecules on particle drag, and these forces were not considered in previous studies (e.g., [3]). The purpose of the present study is to generalize our analysis to particle transport in low-density gases with nonuniform temperature. Our special interest here is the thermophoretic force and velocity. Below we shall provide a brief review about past contributions to the formulations of thermophoretic force and velocity, from the continuum to the free molecule regime.

Thermophoresis describes a phenomenon by which particles suspended in a fluid with nonuniform temperatures are subject to a force in the direction opposite to the temperature gradient [4]. This phenomenon was first described by Tyndall [5], who observed that in a chamber filled with dusty air there existed a spatial region around a hot body free of particles. The force that removes the particles from the hot region is termed the thermophoretic force [6]. The thermophoretic force is often counteracted by the fluid drag on the particle, and in steady state the motion of particles has a constant velocity due to equal thermophoretic and drag forces. This velocity is known as the thermophoretic velocity. Thermophoresis is of major importance in a variety of applications, including aerosol science [7], biology [8], and combustion [9]. For example, a recent study [10] demonstrated that depositing flame-synthesized $\mathrm{TiO}_{2}$ nanocrystals onto a substrate with tailored particle sizes and crystal morphology requires a quantitative knowledge of the thermophoretic velocity. This is especially true for particles a few nanometers in size.

Similar to the drag force, the thermophoretic force is strongly dependent on the Knudsen number $\mathrm{Kn}(\mathrm{Kn}=\lambda / R$, where $\lambda$ is the mean free path of the fluid and $R$ the radius of the particle). In the continuum regime $(\mathrm{Kn} \ll 1)$, Epstein [11] examined the thermophoretic force for a sphere immersed in a fluid. Brock [12] improved on Epstein's solution by considering slip boundary conditions in the continuum derivations. Other attempts were made using the Boltzmann equation as the starting point of the analysis [13-15], but the validity of these approaches remains questionable $[11,16,17]$.

In the free molecule regime $(\mathrm{Kn} \gg 1)$, Waldmann [18] proposed an expression for the thermophoretic force that remains the foundation of modern engineering analysis for thermophoresis. The Waldmann equation is given by

$$
F_{T}=-\frac{8}{15} \sqrt{\frac{2 \pi m_{g}}{k T}} \kappa R^{2} \nabla T,
$$

where $m_{g}$ is the mass of the gas molecule, $k$ is the Boltzmann constant, $T$ is the temperature, and $\kappa$ is the thermal conductivity of the gas. The corresponding thermophoretic velocity is given by

$$
V_{T}=-\frac{\kappa \nabla T}{5(1+\pi \varphi / 8) N k T},
$$

where $\varphi$ is known as the momentum accommodation factor and $N$ is the number density of the gas. A value of 0.9 is traditionally chosen for $\varphi$ [19] based on Millikan's oil droplet experiments [20]. In recent studies $[1,2]$ we demonstrated that $\varphi$ depends on particle size and possibly some other factors. For this reason, we shall term $\varphi$ the momentum accommodation function hereafter. Waldmann's solution was later reproduced by Mason and co-workers [21,22] using a dustygas model that considers both specular and diffuse scattering. Experimental [23-26] and numerical studies [27,28] have shown that for large Knudsen numbers the thermophoretic force and velocity approach the Waldmann solution.

In the transition regime $(\mathrm{Kn} \sim 1)$ the problem is somewhat more difficult. Brock [29] extended the solution in the free molecule regime to the transition regime, while Talbot et al. [30] suggested that Brock's continuum equation [12] could 

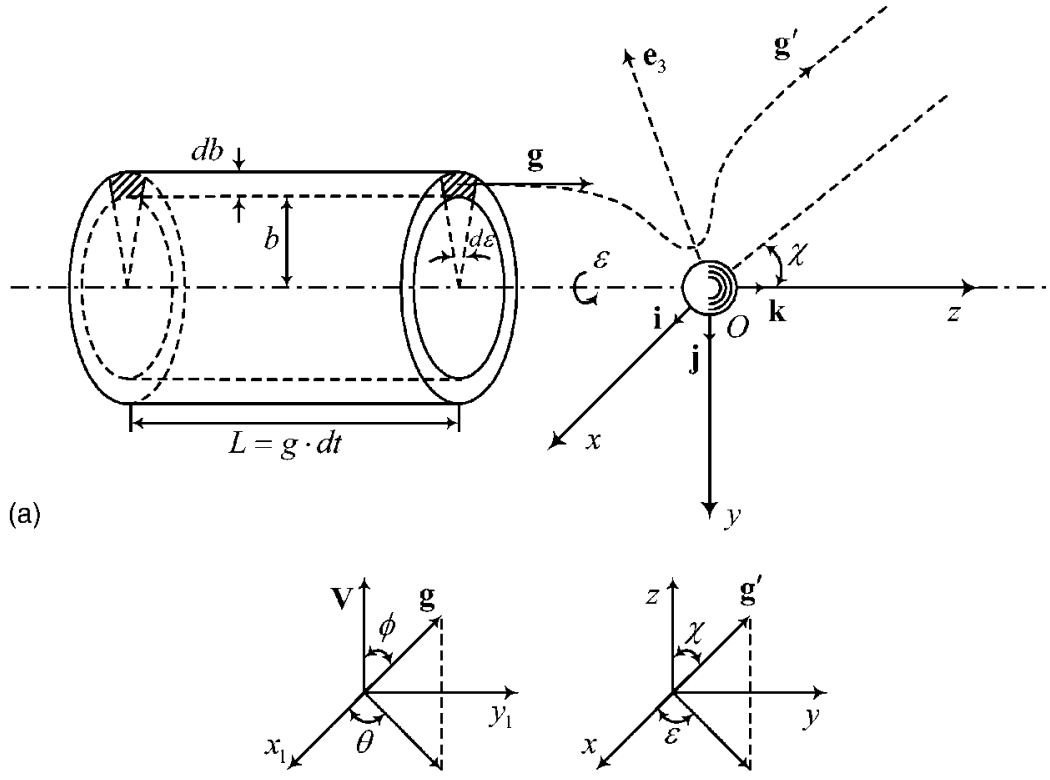

Specular Reflection
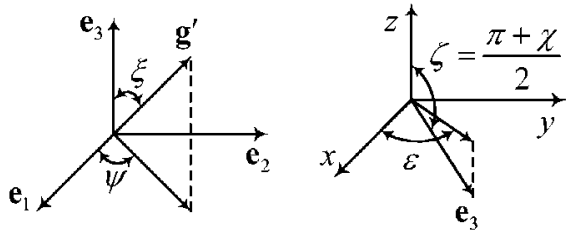

(b)

Diffuse Reflection

be used as an empirical formula for the entire range of $\mathrm{Kn}$ if the coefficients in the formulation were chosen properly. $\mathrm{Nu}-$ merical studies have been reported using several different forms of the Boltzmann equation [27,28,31]. The numerical results seem to agree with experimental data, although questions have been raised concerning the validity of these data since they do not converge to Waldmann's solution at large $\mathrm{Kn}$ [32].

It is seen that the theories for thermophoresis in the continuum and transition regimes are far from complete. In the free molecule regime, however, the gas-kinetic theory appears to work very well as manifested by the success of Waldmann's solution. Yet the accuracy and validity of the Waldmann formulation have not been adequately questioned and tested for nanosized particles. It is possible that based on our previous studies [1,2] the rigid body collision model employed in the Waldmann analysis can lead to large errors for nanosized particles. Furthermore, to accurately predict the thermophoretic velocity, it is crucial that a thermophoretic force formulation be obtained in the manner that is consistent with the drag force formulation reported earlier [1,2].

In this paper, we investigate the thermophoresis of nanoparticles in the free molecule regime. The theoretical foundation of this analysis is the gas-kinetic theory. The influence of van der Waals force and other interactions is considered. It is shown that the current formulas are more general than Waldmann's solution in that these formulas can be reduced to Eq. (1) if a rigid body collision is assumed. The theoretical formulas are then parametrized and presented in a form that can be readily used in applications.
FIG. 1. (a) Collision model and the reference frame. (b) Relationship among various vectors (reproduced from [1]).

\section{A GENERALIZED THEORY FOR FORCES ON A PARTICLE IN THE FREE MOLECULE REGIME}

Following the pioneering work of Epstein [3], we approach the problem by finding the total momentum transfer during collisions between the gas molecules and the particle. The total force exerted on the particle is the net momentum flux upon numerous collisions. Consider a gas in local equilibrium with a temperature gradient given by $\nabla T$. Let the velocity of random motion of a gas molecule relative to the center-of-mass velocity of the gas be $\mathbf{v}$. A particle is introduced into the gas with an instantaneous drift velocity $\mathbf{V}$, again relative to the center-of-mass velocity of the gas. After collision, the velocities of the particle and gas molecule are denoted by $\mathbf{V}^{\prime}$ and $\mathbf{v}^{\prime}$, respectively.

As in our previous study [1], we attach the coordinate system to the particle with the origin $O$ located at the mass center of the particle, as shown in Fig. 1. The velocity of the gas molecules is given by $\mathbf{g}=\mathbf{v}-\mathbf{V}$ before collision and $\mathbf{g}^{\prime}$ $=\mathbf{v}^{\prime}-\mathbf{V}^{\prime}$ after collision. Let the $z$ axis of the coordinate system be parallel to $\mathbf{g}$, and $\mathbf{i}, \mathbf{j}$, and $\mathbf{k}$ be the unit vectors in the $x, y$, and $z$ coordinates, respectively.

Consider the gas molecules traveling in a cylindrical region with an impact parameter $b$ and velocity g (Fig. 1) and a small sector of this cylindrical shell of an area given by $b d b d \varepsilon$. In the coordinate system defined above, the differential force in the sector on the particle $d \mathbf{F}$ is the time derivative of the momentum of gas molecules, 


$$
d \mathbf{F}=\frac{\mathbf{p}-\mathbf{p}^{\prime}}{d t}
$$

where $\mathbf{p}$ and $\mathbf{p}^{\prime}$ denote the momenta before and after collision, respectively. The incident momentum $\mathbf{p}$ for the molecules in the sector is given by

$$
\mathbf{p}=m_{r} n \mathbf{g},
$$

where $n$ is the number of molecules in this sector at time $t$,

$$
n=f g b d b d \varepsilon d t,
$$

$m_{r}=m_{g} m_{p} /\left(m_{g}+m_{p}\right)$ is the reduced mass of the gas molecule and a particle with mass equal to $m_{p}$, and $f$ is the velocity distribution function of the gas molecules. In the presence of a thermal gradient, the velocity distribution function is given by $[33,34]$

$$
f=f^{0}\left(1-f^{T}\right)
$$

where $f^{0}$ is the velocity distribution function with uniform temperature distribution

$$
f^{0}=\frac{N}{\left(2 \pi k T / m_{r}\right)^{3 / 2}} e^{-v^{2} /\left(2 k T / m_{r}\right)},
$$

and $f^{T}$ accounts for the temperature variation,

$$
f^{T}=\frac{2 m_{r} \kappa}{5 N(k T)^{2}}\left(\frac{m_{r} v^{2}}{2 k T}-\frac{5}{2}\right) \frac{\mathbf{V} \cdot \mathbf{v}}{V} \nabla T .
$$

The momentum of reflected molecules $\mathbf{p}^{\prime}$ depends on the scattering model. We shall treat here separately two limiting models of collision, namely, specular and diffuse scattering. For specular scattering, the angle of incidence is equal to that of reflection, as shown in Fig. 1, and the magnitudes of $\mathbf{g}$ and $\mathbf{g}^{\prime}$ are assumed to be equal. For diffuse scattering, the magnitude of $\mathbf{g}^{\prime}$ is Maxwellian, and the direction of $\mathbf{g}^{\prime}$ is random above the element surface of impact [20,35]. The dynamics of flyby scattering are identical for both cases. [36]

For specular scattering the angle of scattering is given by

$$
\chi(g, b)=\pi-2 b \int_{r_{m}}^{\infty} r^{-2}\left[1-\frac{b^{2}}{r^{2}}-\frac{\Phi(r)}{m_{r} g^{2} / 2}\right]^{-1 / 2} d r,
$$

where $r$ is the center-to-center distance between the gas molecule and particle, and $r_{m}$ is the distance of the closest encounter. The van der Waals and other forces between the gas molecule and particle are accounted for by $\Phi(r)$, i.e., the potential function of interactions. The momentum of the reflected molecules is therefore

$$
\mathbf{p}^{\prime}=m_{r} n g(\mathbf{k} \cos \chi+\mathbf{i} \sin \chi \cos \varepsilon+\mathbf{j} \sin \chi \sin \varepsilon) .
$$

It is shown previously [1] that the second and third terms in the above equation vanish upon integration over $\varepsilon$ from 0 to $2 \pi$, and thus only the first term needs to be considered. Combining Eqs. (3) through (6) and (10) and integrating over $\mathbf{v}$, $b$, and $\varepsilon$, we obtain the force in the limit of specular scattering $\mathbf{F}_{s}$ as

$$
\mathbf{F}_{s}=m_{r} \int_{\mathbf{v}} g \mathbf{g}\left(f^{0}-f^{0} f^{T}\right) Q_{s}(g) d \mathbf{v}
$$

where $Q_{s}(g)$ is the collision cross section [36],

$$
Q_{s}(g)=2 \pi \int_{0}^{\infty}(1-\cos \chi) b d b .
$$

Clearly, the force in Eq. (11) can be split into two terms such that $\mathbf{F}_{s}=\mathbf{F}_{D, s}+\mathbf{F}_{T, s}$, where the first term $\mathbf{F}_{D, s}$ is the drag,

$$
\mathbf{F}_{D, s}=m_{r} \int_{\mathbf{v}} g \mathbf{g} f^{0} Q_{s}(g) d \mathbf{v}
$$

and the second term accounts for the thermophoretic force,

$$
\mathbf{F}_{T, s}=-m_{r} \int_{\mathbf{v}} g \mathbf{g} f^{0} f^{T} Q_{s}(g) d \mathbf{v}
$$

The solution of Eq. (13) has been obtained previously [1] as

$$
\mathbf{F}_{D, s}=-\frac{8}{3} \sqrt{2 \pi m_{r} k T} N R^{2} \Omega_{s}^{(1,1)^{*}} \mathbf{V}
$$

where $\Omega_{s}^{(1, l)^{*}}$ is the reduced collision integral [36]

$$
\Omega_{s}^{(1, l) *}=\frac{2 \int_{0}^{\infty} \gamma^{2 l+3} e^{-\gamma^{2}} Q_{s}(g) d \gamma}{(l+1) ! \pi R^{2}},
$$

and $\gamma=g / \sqrt{2 k T / m_{r}}$. For the thermophoretic force, we combine Eq. (14) with Eqs. (7) and (8) and simplify to obtain

$$
\begin{aligned}
\mathbf{F}_{T, s}= & -\frac{8}{5 \pi^{3 / 2}}\left(\frac{m_{r}}{2 k T}\right)^{7 / 2} \kappa \nabla T \mathbf{u} \int_{\mathbf{v}} g \mathbf{g} e^{-v^{2} /\left(2 k T / m_{r}\right)} \\
& \times\left(\frac{m_{r} v^{2}}{2 k T}-\frac{5}{2}\right) \mathbf{v} Q_{s}(g) d \mathbf{v},
\end{aligned}
$$

where $\mathbf{u}=\mathbf{V} / V$ is the unit vector in the direction of the drift velocity of the particle. As in the previous study [1], we assume that $V \ll v$, because the particle is much heavier than a gas molecule yet the temperatures (kinetic energies) of the particle and gas are not very different from each other. The error resulting from this assumption will be discussed in Sec. $\mathrm{V}$. We therefore have $d \mathbf{v} \approx d \mathbf{g}$. Let $\phi$ and $\theta$ be the colatitude and azimuthal angles of $\mathbf{g}$ in a reference frame in which $\mathbf{V}$ is collinear with the $z$ axis (Fig. 1). We have $v^{2}=g^{2}+V^{2}$ $+2 g V \cos \phi \approx g^{2}+2 g V \cos \phi$ for $g \gg V$. Since $k T \sim m_{g} g^{2}$ $\sim m_{r} g^{2} \gg m_{r} g V, g V /\left(k T / m_{r}\right) \ll 1$. Therefore the exponential term in Eq. (17) may be expanded and simplified to

$$
e^{-\left(g^{2}+2 g V \cos \phi\right) /\left(2 k T / m_{r}\right)} \approx\left(1-\frac{g V \cos \phi}{k T / m_{r}}\right) e^{-g^{2} /\left(2 k T / m_{r}\right)} \approx e^{-\gamma^{2}} .
$$

Furthermore, it can be shown that 


$$
\begin{aligned}
{\left[\frac{m_{r} v^{2}}{2 k T}-\frac{5}{2}\right] \mathbf{u} \cdot \mathbf{v} \approx } & \left(\gamma^{2}-\frac{5}{2}\right)\left(\gamma \sqrt{2 k T / m_{r}} \cos \phi+V\right) \\
& +2 \gamma^{2} V \cos ^{2} \phi .
\end{aligned}
$$

Using Eqs. (18) and (19) and $g=\gamma \sqrt{2 k T / m_{r}}$, we rewrite Eq. (17) as

$$
\begin{aligned}
\mathbf{F}_{T, s}= & -\frac{16}{5 \sqrt{\pi}}\left(\frac{m_{r}}{2 k T}\right) \kappa \nabla T \mathbf{u} \int_{0}^{\infty} \int_{0}^{\pi} \gamma^{4} e^{-\gamma^{2}}\left[\left(\gamma^{2}-\frac{5}{2}\right)\right. \\
& \left.\times\left(\gamma \sqrt{2 k T / m_{r}} \cos \phi+V\right)+2 \gamma^{2} V \cos ^{2} \phi\right] \\
& \times Q_{s}(g) \sin \phi \cos \phi d \phi d \gamma .
\end{aligned}
$$

Eliminating the inner integral, we obtain

$$
\begin{aligned}
\mathbf{F}_{T, s}= & \frac{8}{3} \sqrt{\frac{2 m_{r}}{\pi k T}} \kappa \nabla T\left[\int_{0}^{\infty} \gamma^{5} e^{-\gamma^{2}} Q_{s}(\gamma) d \gamma\right. \\
& \left.-\frac{2}{5} \int_{0}^{\infty} \gamma^{7} e^{-\gamma^{2}} Q_{s}(\gamma) d \gamma\right] \mathbf{u} .
\end{aligned}
$$

The above equation may be expressed in terms of the reduced collision integrals $\Omega_{s}^{(1,1)^{*}}$ and $\Omega_{s}^{(1,2)^{*}}$ as

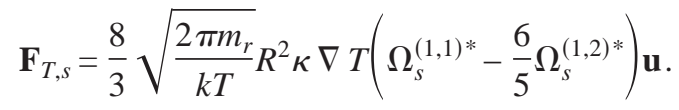

We note that $\Omega_{s}^{(1,1) *}-6 \Omega_{s}^{(1,2) *} / 5<0$. Therefore the unit vector $\mathbf{u}$ in Eq. (22) ensures that the thermophoretic force is in the direction opposite to the temperature gradient. The interactions of gas molecules and particle are manifested in the reduced collision integrals. It can be shown that in the limit of rigid body collision $\left(\Omega_{s}^{(1,1)^{*}}=1\right.$ and $\left.\Omega_{s}^{(1,2)^{*}}=1\right)$ and assuming that $m_{p} \gg m_{g}$, Eq. (22) is easily reduced to the Waldmann equation (1).

We shall now obtain a similar expression for diffuse scattering. The velocity distribution of molecules diffusely scattered is Maxwellian and given by [1]

$$
f^{\prime}=\frac{1}{2 \pi}\left(\frac{m_{r}}{k T}\right)^{2} g f^{0}\left(1-f^{T}\right) e^{-g^{\prime 2} /\left(2 k T / m_{r}\right)} .
$$

The subsequent analysis is simplified by recognizing that the effective angle of diffuse scattering is normal to the surface of impact [1], because the net momentum transfer is zero in the direction parallel to the surface. Define the polar angles between $\mathbf{g}^{\prime}$ and $\mathbf{e}_{3}$ as $\xi$ and $\psi$, where $\mathbf{e}_{3}$ is a unit vector as shown in Fig. 1. The momentum of the reflected molecules can be written as

$$
\begin{aligned}
\mathbf{p}^{\prime} & =b d b d \varepsilon d t \int_{\mathbf{g}^{\prime}} m_{r} g^{\prime} \mathbf{g}^{\prime} \cos \xi f^{\prime} d \mathbf{g}^{\prime} \\
& =\frac{m_{r} n \mathbf{e}_{3}}{\left(k T / m_{r}\right)^{2}} \int_{0}^{\infty} g^{\prime 4} e^{-g^{\prime 2} /\left(2 k T / m_{r}\right)} d g^{\prime} \int_{0}^{\pi / 2} \cos ^{2} \xi \sin \xi d \xi .
\end{aligned}
$$

Integrating the above equation, we find that

$$
\mathbf{p}^{\prime}=\sqrt{\frac{\pi m_{r} k T}{2}} n \mathbf{e}_{3}=\sqrt{\frac{\pi m_{r} k T}{2}} g f b d b d \varepsilon d t \mathbf{e}_{3} .
$$

Decomposing the unit vector $\mathbf{e}_{3}$ on the principal axes of the coordinate system, we obtain the momentum of the reflected molecule as

$$
\begin{aligned}
\mathbf{p}^{\prime}= & \sqrt{\frac{\pi m_{r} k T}{2}}[\mathbf{g} \cos \zeta+\mathbf{i} g \sin \zeta \cos \varepsilon \\
& +\mathbf{j} g \sin \zeta \sin \varepsilon] f b d b d \varepsilon d t,
\end{aligned}
$$

where $\zeta=(\chi+\pi) / 2$. Putting Eqs. (26) and (4) into Eq. (3) and integrating, we obtain the force equation as

$$
\mathbf{F}_{d}=m_{r} \int_{\mathbf{v}} g \mathbf{g} f^{0}\left(1-f^{T}\right) Q_{d}(g) d \mathbf{v},
$$

where $Q_{d}(g)$ is the diffuse scattering cross section

$$
Q_{d}(g)=2 \pi \int_{0}^{\infty}\left(1+\frac{1}{g} \sqrt{\frac{\pi k T}{2 m_{r}}} \sin \frac{\chi}{2}\right) b d b .
$$

The above integral is divergent and should be modified to account for the fact that for $b \geqslant \mathrm{a}$ critical impact factor $b_{0}$, diffuse scattering is switched to orbiting $\left(b=b_{0}\right)$ and grazing $\left(b>b_{0}\right)$ scattering [1,36] (see Fig. 3 of [1]). The resulting expression is

$$
\begin{aligned}
Q_{d}(g)= & 2 \pi\left[\int_{0}^{b_{0}}\left(1+\frac{1}{g} \sqrt{\frac{\pi k T}{2 m_{r}}} \sin \frac{\chi}{2}\right) b d b\right. \\
& \left.+\int_{b_{0}}^{\infty}(1-\cos \chi) b d b\right] .
\end{aligned}
$$

Again Eq. (27) can be split into a drag force term and a thermophoretic force term,

$$
\mathbf{F}_{d}=\mathbf{F}_{D, d}+\mathbf{F}_{T, d}=m_{r} \int_{\mathbf{v}} g \mathbf{g} f^{0} Q_{d}(g) d \mathbf{v}-m_{r} \int_{\mathbf{v}} g \mathbf{g} f^{0} f^{T} Q_{d}(g) d \mathbf{v},
$$

where $\mathbf{F}_{D, d}=-(8 / 3) \sqrt{2 \pi m_{r} k T} N R^{2} \Omega_{d}^{(1,1) *} \mathbf{V}$ [1]. For the thermophoretic term, we obtain the force equation in a similar manner as that for specular scattering,

$$
\mathbf{F}_{T, d}=\frac{8}{3} \sqrt{\frac{2 \pi m_{r}}{k T}} R^{2} \kappa \nabla T\left(\Omega_{d}^{(1,1)^{*}}-\frac{6}{5} \Omega_{d}^{(1,2) *}\right) \mathbf{u},
$$

where $\Omega_{d}^{(1,1)^{*}}$ and $\Omega_{d}^{(1,2)^{*}}$ are the reduced diffuse scattering collision integrals

$$
\Omega_{d}^{(1, l) *}=\frac{2 \int_{0}^{\infty} \gamma^{2 l+3} e^{-\gamma^{2}} Q_{d}(g) d \gamma}{(l+1) ! \pi R^{2}} .
$$

Comparing the specular and diffuse scattering models, we see that the formulations governing the thermophoretic force and reduced collision integral are identical. The difference is manifested by the collision cross section [see Eqs. (12) and (29)]. This difference, however, vanishes when the rigid body collision model is adopted. Specifically, the collision integrals in Eq. (32) are simplified to $\Omega_{d}^{(1,1)^{*}}=1+\pi / 8$ and 
$\Omega_{d}^{(1,2) *}=1+5 \pi / 48$ for rigid body collision. Then again, Eq. (31) turns out to be the exact Waldmann solution. It has been shown [2] that the rigid body collision is accurate as long as the particles are much larger than $1 \mathrm{~nm}$. Therefore, for large particles the choice of the collision model (i.e., specular and diffuse scattering) is inconsequential to the thermophoretic force.

\section{PARAMETRIZATION}

The derivations in the preceding section consider two limiting scattering cases. The real case should fall between these limits [2]. A generalized equation for thermophoretic force may be obtained in terms of average reduced collision integrals $\Omega_{\text {avg }}^{(1,1) *}$ and $\Omega_{\text {avg }}^{(1,2) *}$ and parametrized by

$$
\Omega_{a v g}^{(1, l)^{*}}=\varphi \Omega_{d}^{(1, l) *}+(1-\varphi) \Omega_{s}^{(1, l) *},
$$

where $l=1$ or 2 , and $\varphi$ is the momentum accommodation function to be discussed in Sec. IV. This parametrization accounts for the limiting case of diffuse scattering with $\varphi=1$ and of specular scattering with $\varphi=0$. The resulting thermophoretic force may be written in terms of $\Omega_{a v g}^{(1, l)^{*}}$ as

$$
\mathbf{F}_{T}=\frac{8}{3} \sqrt{\frac{2 \pi m_{r}}{k T}} R^{2} \kappa \nabla T\left(\Omega_{\text {avg }}^{(1,1)^{*}}-\frac{6}{5} \Omega_{\text {avg }}^{(1,2) *}\right) \mathbf{u},
$$

and the drag force is

$$
\mathbf{F}_{D}=-\frac{8}{3} \sqrt{2 \pi m_{r} k T N} N R^{2} \Omega_{a v g}^{(1,1) *} \mathbf{V} .
$$

\section{COLLISION INTEGRALS AND MOMENTUM ACCOMMODATION FUNCTION}

The collision integrals $\Omega^{(1,1)^{*}}$ for both specular and diffuse scattering are tabulated in [1] using RudyakKrasnolutski's 9-3 potential function [37]. Here we shall tabulate $\Omega^{(1,2) *}$ values. In its original form the RudyakKrasnolutski potential energy is expressed as a function of the center-to-center separation distance $r$ as

$$
\Phi(r)=\Phi_{9}(r)-\Phi_{3}(r),
$$

where $\quad \Phi_{i}(r)=C_{i}\left\{\left[(r-R)^{-i}-(r+R)^{-i}\right]-a_{i}\left[(r-R)^{-i+1}-(r\right.\right.$ $\left.\left.+R)^{-i+1}\right]\right\}, a_{9}=9 r / 8, a_{3}=3 r / 2, C_{9}=4 \pi \varepsilon \sigma^{12} /(45 \nu)$, and $C_{3}$ $=2 \pi \varepsilon \sigma^{6} /(3 \nu), \nu=\bar{M} / \rho_{s}$ is the effective volume of the particle per molecule, $\rho_{s}$ is the particle mass density, and $\bar{M}$ is the mean atomic mass of the particle material. The collision diameter $\sigma$ and well depth $\varepsilon$ are those of the Lennard-Jones (12-6) potential function for the interactions of the gas molecule and constituent atom or molecule of the particle. These potential energy parameters may be estimated from the selfinteraction potential parameters $[2,37]$ using the simple mixing rules $\sigma=\left(\sigma_{g}+\sigma_{p}\right) / 2$ and $\varepsilon=\sqrt{\varepsilon_{g} \varepsilon_{p}}$, where $g$ and $p$ denote the gas molecule and constituent atom or molecule of the particle.

Equation (36) may be nondimensionalized [2] and expressed as a function of modified reduced temperature $T^{*}$ $=k T / \varepsilon^{\prime}$ and reduced collision diameter $\sigma^{\prime}=\sigma / R$ with $\varepsilon^{\prime}$ $=2 \pi \varepsilon \sigma^{3} / 3 \nu$. It follows that $\Omega^{(1,2)^{*}}$ may be tabulated as a function of $T^{*}$ and $\sigma^{\prime}$ as presented in Tables I and II for specular and diffuse scattering, respectively. The ranges $0.1<T^{*}<100$ and $0.01<\sigma^{\prime}<0.6$ are chosen to be sufficiently wide for all practical purposes. For example, the maximum $\sigma^{\prime}$ value ensures that the collision integral is applicable to particles with radius as small as $0.5 \mathrm{~nm}$.

These same $\Omega^{(1,2) *}$ values are plotted in Figs. 2 and 3 as a function of $T^{*}$ and $\sigma^{\prime}$. It is seen that just like $\Omega^{(1,1)^{*}}$ [2], toward high temperatures and large particle size (small $\sigma^{\prime}$ ), the $\Omega^{(1,2)^{*}}$ integrals approach their asymptotic limits of 1 and $1+5 \pi / 48$ for specular and diffuse scattering, respectively. The influence of nonrigid body collision occurs mostly for low temperatures and/or small particle sizes.

We parametrize the reduced collision integrals in the form of [2]

$$
\begin{aligned}
\Omega_{s / d}^{(1,2)^{*}}= & 1+a+\left[a_{T_{0}}+\frac{a_{T_{1}}}{T^{* 1 / 4}}+\frac{a_{T_{2}}}{T^{* 1 / 2}}\right] \sigma^{\prime} \\
& +\left[b_{T_{0}}-\frac{b_{T_{1}}}{T^{* 1 / 4}}+\frac{b_{T_{2}}}{T^{* 1 / 2}}\right] \sigma^{\prime 2},
\end{aligned}
$$

where the $a$ 's and $b$ 's are coefficients, and their values are found in Table III. Equation (37) satisfies the asymptotic limits of rigid body collision at $T * \rightarrow \infty$ and/or $\sigma^{\prime} \rightarrow 0$. The maximum fitting errors are $3.9 \%$ and $3.0 \%$ for $\Omega_{d}^{(1,2) *}$ and $\Omega_{s}^{(1,2) *}$, respectively, both of which occur for large $\sigma^{\prime}$ and small $T^{*}$, i.e., the high-curvature areas shown in Figs. 2 and 3. In other regions, the fitting error is well within $2 \%$.

As discussed in [2], the momentum accommodation function $\varphi$ in Eq. (33) is dependent on the particle size. By comparing the particle radius measured by transmission electronic microscopy with mobility size data measured at room temperature and ambient pressure, we found that $\varphi$ switches from a value of 0 to a value of 0.9 at $2-3 \mathrm{~nm}$ in particle radius (see, for example, Fig. 7 of [2]). This function was empirically fitted in the following form:

$$
\varphi=\frac{1+0.9 \mathrm{Kn}\left\{1-1 /\left[1+(R / 2.5)^{15}\right]\right\}}{1+\mathrm{Kn}},
$$

where $R$ is in nanometers. Equation (38) accounts for the transition from specular-scattering -dominated collision dynamics for particles with sizes approaching the molecular size [33] to diffuse scattering being the predominant collision outcome for large particles as reported by Millikan [20]. Putting Eq. (38) into Eq. (33), we obtain the average reduced collision integral as 
TABLE I. Reduced collision integral $\Omega_{s}^{(1,2) *}$.

\begin{tabular}{|c|c|c|c|c|c|c|c|c|c|c|c|c|c|c|c|}
\hline \multirow[b]{2}{*}{$T^{*}$} & \multicolumn{15}{|c|}{$\sigma^{\prime}$} \\
\hline & 0.01 & 0.03 & 0.05 & 0.07 & 0.09 & 0.11 & 0.13 & 0.15 & 0.17 & 0.19 & 0.2 & 0.3 & 0.4 & 0.5 & 0.6 \\
\hline 0.10 & 1.04 & 1.13 & 1.21 & 1.31 & 1.38 & 1.49 & 1.59 & 1.66 & 1.75 & 1.88 & 1.92 & 2.50 & 3.21 & 3.96 & 4.75 \\
\hline 0.15 & 1.04 & 1.11 & 1.18 & 1.27 & 1.34 & 1.42 & 1.52 & 1.58 & 1.65 & 1.76 & 1.80 & 2.28 & 2.86 & 3.49 & 4.12 \\
\hline 0.20 & 1.04 & 1.10 & 1.17 & 1.24 & 1.31 & 1.38 & 1.47 & 1.53 & 1.59 & 1.69 & 1.72 & 2.15 & 2.66 & 3.20 & 3.75 \\
\hline 0.25 & 1.03 & 1.09 & 1.15 & 1.22 & 1.29 & 1.35 & 1.44 & 1.50 & 1.55 & 1.64 & 1.67 & 2.06 & 2.52 & 3.00 & 3.48 \\
\hline 0.30 & 1.03 & 1.09 & 1.15 & 1.21 & 1.27 & 1.33 & 1.41 & 1.47 & 1.52 & 1.60 & 1.63 & 2.00 & 2.41 & 2.85 & 3.29 \\
\hline 0.35 & 1.03 & 1.09 & 1.14 & 1.20 & 1.26 & 1.31 & 1.39 & 1.44 & 1.50 & 1.57 & 1.60 & 1.95 & 2.33 & 2.73 & 3.13 \\
\hline 0.40 & 1.03 & 1.08 & 1.13 & 1.19 & 1.25 & 1.30 & 1.37 & 1.43 & 1.48 & 1.55 & 1.57 & 1.90 & 2.26 & 2.63 & 2.99 \\
\hline 0.45 & 1.03 & 1.08 & 1.13 & 1.18 & 1.24 & 1.29 & 1.36 & 1.41 & 1.46 & 1.53 & 1.55 & 1.87 & 2.20 & 2.54 & 2.88 \\
\hline 0.50 & 1.03 & 1.07 & 1.12 & 1.18 & 1.23 & 1.28 & 1.34 & 1.40 & 1.45 & 1.51 & 1.54 & 1.84 & 2.15 & 2.47 & 2.79 \\
\hline 0.55 & 1.02 & 1.07 & 1.12 & 1.17 & 1.22 & 1.27 & 1.33 & 1.38 & 1.44 & 1.49 & 1.52 & 1.81 & 2.10 & 2.40 & 2.71 \\
\hline 0.60 & 1.02 & 1.07 & 1.12 & 1.17 & 1.22 & 1.26 & 1.32 & 1.37 & 1.43 & 1.48 & 1.51 & 1.78 & 2.06 & 2.35 & 2.64 \\
\hline 0.65 & 1.02 & 1.07 & 1.11 & 1.16 & 1.21 & 1.26 & 1.31 & 1.37 & 1.42 & 1.47 & 1.50 & 1.76 & 2.03 & 2.30 & 2.57 \\
\hline 0.70 & 1.02 & 1.07 & 1.11 & 1.16 & 1.21 & 1.25 & 1.31 & 1.36 & 1.41 & 1.46 & 1.49 & 1.74 & 1.99 & 2.25 & 2.52 \\
\hline 0.75 & 1.02 & 1.06 & 1.11 & 1.16 & 1.20 & 1.25 & 1.30 & 1.35 & 1.40 & 1.45 & 1.47 & 1.72 & 1.97 & 2.22 & 2.47 \\
\hline 0.80 & 1.02 & 1.06 & 1.11 & 1.15 & 1.20 & 1.24 & 1.29 & 1.34 & 1.39 & 1.44 & 1.47 & 1.70 & 1.94 & 2.18 & 2.43 \\
\hline 0.85 & 1.02 & 1.06 & 1.11 & 1.15 & 1.19 & 1.24 & 1.29 & 1.34 & 1.39 & 1.43 & 1.46 & 1.69 & 1.92 & 2.15 & 2.39 \\
\hline 0.90 & 1.02 & 1.06 & 1.11 & 1.15 & 1.19 & 1.24 & 1.28 & 1.33 & 1.38 & 1.42 & 1.45 & 1.67 & 1.89 & 2.12 & 2.36 \\
\hline 0.95 & 1.02 & 1.06 & 1.11 & 1.15 & 1.19 & 1.23 & 1.28 & 1.33 & 1.37 & 1.41 & 1.44 & 1.66 & 1.87 & 2.10 & 2.33 \\
\hline 1.00 & 1.02 & 1.06 & 1.10 & 1.14 & 1.19 & 1.23 & 1.27 & 1.32 & 1.37 & 1.40 & 1.43 & 1.64 & 1.86 & 2.07 & 2.30 \\
\hline 1.10 & 1.02 & 1.06 & 1.10 & 1.14 & 1.18 & 1.22 & 1.27 & 1.31 & 1.36 & 1.39 & 1.42 & 1.62 & 1.82 & 2.03 & 2.25 \\
\hline 1.20 & 1.02 & 1.06 & 1.10 & 1.14 & 1.18 & 1.22 & 1.26 & 1.30 & 1.35 & 1.38 & 1.41 & 1.60 & 1.80 & 2.00 & 2.21 \\
\hline 1.30 & 1.02 & 1.05 & 1.10 & 1.13 & 1.18 & 1.21 & 1.25 & 1.29 & 1.34 & 1.37 & 1.40 & 1.58 & 1.77 & 1.97 & 2.18 \\
\hline 1.40 & 1.02 & 1.05 & 1.10 & 1.13 & 1.17 & 1.21 & 1.25 & 1.29 & 1.33 & 1.36 & 1.39 & 1.57 & 1.75 & 1.95 & 2.15 \\
\hline 1.50 & 1.02 & 1.05 & 1.10 & 1.13 & 1.17 & 1.20 & 1.24 & 1.28 & 1.32 & 1.35 & 1.38 & 1.55 & 1.74 & 1.92 & 2.12 \\
\hline 1.60 & 1.02 & 1.05 & 1.09 & 1.12 & 1.17 & 1.20 & 1.24 & 1.28 & 1.31 & 1.35 & 1.37 & 1.54 & 1.72 & 1.91 & 2.10 \\
\hline 1.70 & 1.02 & 1.05 & 1.09 & 1.12 & 1.16 & 1.20 & 1.24 & 1.27 & 1.31 & 1.34 & 1.36 & 1.53 & 1.71 & 1.89 & 2.08 \\
\hline 1.80 & 1.02 & 1.05 & 1.09 & 1.12 & 1.16 & 1.19 & 1.23 & 1.27 & 1.30 & 1.33 & 1.35 & 1.52 & 1.69 & 1.87 & 2.06 \\
\hline 1.90 & 1.02 & 1.05 & 1.09 & 1.12 & 1.16 & 1.19 & 1.23 & 1.26 & 1.30 & 1.33 & 1.35 & 1.51 & 1.68 & 1.86 & 2.04 \\
\hline 2.00 & 1.02 & 1.05 & 1.09 & 1.12 & 1.16 & 1.19 & 1.22 & 1.26 & 1.29 & 1.32 & 1.34 & 1.50 & 1.67 & 1.85 & 2.03 \\
\hline 3.00 & 1.02 & 1.05 & 1.08 & 1.11 & 1.14 & 1.17 & 1.20 & 1.23 & 1.26 & 1.29 & 1.30 & 1.45 & 1.61 & 1.77 & 1.94 \\
\hline 4.00 & 1.01 & 1.05 & 1.07 & 1.10 & 1.13 & 1.16 & 1.18 & 1.21 & 1.24 & 1.27 & 1.28 & 1.42 & 1.57 & 1.72 & 1.88 \\
\hline 5.00 & 1.01 & 1.05 & 1.07 & 1.10 & 1.12 & 1.15 & 1.18 & 1.20 & 1.23 & 1.26 & 1.27 & 1.41 & 1.55 & 1.70 & 1.85 \\
\hline 6.00 & 1.01 & 1.04 & 1.06 & 1.09 & 1.12 & 1.14 & 1.17 & 1.19 & 1.22 & 1.25 & 1.26 & 1.39 & 1.53 & 1.68 & 1.83 \\
\hline 7.00 & 1.01 & 1.04 & 1.06 & 1.09 & 1.11 & 1.14 & 1.16 & 1.19 & 1.21 & 1.24 & 1.25 & 1.38 & 1.52 & 1.66 & 1.81 \\
\hline 8.00 & 1.01 & 1.04 & 1.06 & 1.09 & 1.11 & 1.14 & 1.16 & 1.18 & 1.21 & 1.23 & 1.25 & 1.37 & 1.51 & 1.65 & 1.79 \\
\hline 9.00 & 1.01 & 1.04 & 1.06 & 1.09 & 1.11 & 1.13 & 1.16 & 1.18 & 1.20 & 1.23 & 1.24 & 1.37 & 1.50 & 1.64 & 1.78 \\
\hline 10.00 & 1.01 & 1.04 & 1.06 & 1.08 & 1.11 & 1.13 & 1.15 & 1.18 & 1.20 & 1.23 & 1.24 & 1.36 & 1.49 & 1.63 & 1.77 \\
\hline 20.00 & 1.01 & 1.03 & 1.05 & 1.07 & 1.10 & 1.12 & 1.14 & 1.16 & 1.18 & 1.20 & 1.22 & 1.33 & 1.45 & 1.57 & 1.70 \\
\hline 30.00 & 1.01 & 1.03 & 1.05 & 1.07 & 1.09 & 1.11 & 1.13 & 1.15 & 1.17 & 1.19 & 1.20 & 1.31 & 1.42 & 1.54 & 1.66 \\
\hline 40.00 & 1.01 & 1.03 & 1.05 & 1.07 & 1.09 & 1.11 & 1.13 & 1.15 & 1.17 & 1.19 & 1.20 & 1.30 & 1.41 & 1.52 & 1.64 \\
\hline 50.00 & 1.01 & 1.03 & 1.05 & 1.06 & 1.08 & 1.10 & 1.12 & 1.14 & 1.16 & 1.18 & 1.19 & 1.29 & 1.40 & 1.51 & 1.62 \\
\hline 60.00 & 1.01 & 1.03 & 1.05 & 1.06 & 1.08 & 1.10 & 1.12 & 1.14 & 1.16 & 1.18 & 1.19 & 1.29 & 1.39 & 1.50 & 1.61 \\
\hline 70.00 & 1.01 & 1.03 & 1.04 & 1.06 & 1.08 & 1.10 & 1.12 & 1.14 & 1.15 & 1.17 & 1.18 & 1.28 & 1.38 & 1.49 & 1.59 \\
\hline 80.00 & 1.01 & 1.03 & 1.04 & 1.06 & 1.08 & 1.10 & 1.12 & 1.13 & 1.15 & 1.17 & 1.18 & 1.28 & 1.38 & 1.48 & 1.58 \\
\hline 90.00 & 1.01 & 1.03 & 1.04 & 1.06 & 1.08 & 1.10 & 1.11 & 1.13 & 1.15 & 1.17 & 1.18 & 1.27 & 1.37 & 1.47 & 1.58 \\
\hline 100.00 & 1.01 & 1.03 & 1.04 & 1.06 & 1.08 & 1.09 & 1.11 & 1.13 & 1.15 & 1.17 & 1.18 & 1.27 & 1.36 & 1.46 & 1.57 \\
\hline
\end{tabular}


TABLE II. Reduced collision integral $\Omega_{d}^{(1,2) *}$.

\begin{tabular}{|c|c|c|c|c|c|c|c|c|c|c|c|c|c|c|c|}
\hline \multirow[b]{2}{*}{$T^{*}$} & \multicolumn{15}{|c|}{$\sigma^{\prime}$} \\
\hline & 0.01 & 0.03 & 0.05 & 0.07 & 0.09 & 0.11 & 0.13 & 0.15 & 0.17 & 0.19 & 0.2 & 0.3 & 0.4 & 0.5 & 0.6 \\
\hline 0.10 & 1.44 & 1.60 & 1.74 & 1.89 & 2.04 & 2.18 & 2.33 & 2.48 & 2.63 & 2.79 & 2.87 & 3.70 & 4.63 & 5.65 & 6.75 \\
\hline 0.15 & 1.42 & 1.57 & 1.70 & 1.83 & 1.96 & 2.08 & 2.21 & 2.34 & 2.47 & 2.60 & 2.67 & 3.38 & 4.16 & 5.01 & 5.92 \\
\hline 0.20 & 1.41 & 1.55 & 1.67 & 1.79 & 1.91 & 2.02 & 2.13 & 2.25 & 2.37 & 2.49 & 2.55 & 3.19 & 3.88 & 4.62 & 5.42 \\
\hline 0.25 & 1.41 & 1.54 & 1.65 & 1.76 & 1.87 & 1.97 & 2.08 & 2.19 & 2.30 & 2.41 & 2.46 & 3.05 & 3.68 & 4.35 & 5.07 \\
\hline 0.30 & 1.41 & 1.53 & 1.63 & 1.74 & 1.84 & 1.94 & 2.04 & 2.14 & 2.25 & 2.35 & 2.40 & 2.95 & 3.53 & 4.14 & 4.80 \\
\hline 0.35 & 1.40 & 1.52 & 1.62 & 1.72 & 1.82 & 1.91 & 2.01 & 2.10 & 2.21 & 2.30 & 2.35 & 2.87 & 3.42 & 3.98 & 4.58 \\
\hline 0.40 & 1.40 & 1.51 & 1.61 & 1.70 & 1.80 & 1.89 & 1.98 & 2.07 & 2.17 & 2.26 & 2.31 & 2.80 & 3.32 & 3.84 & 4.40 \\
\hline 0.45 & 1.40 & 1.51 & 1.60 & 1.69 & 1.78 & 1.87 & 1.96 & 2.05 & 2.14 & 2.23 & 2.28 & 2.75 & 3.23 & 3.73 & 4.25 \\
\hline 0.50 & 1.40 & 1.50 & 1.59 & 1.68 & 1.77 & 1.85 & 1.94 & 2.03 & 2.12 & 2.20 & 2.25 & 2.70 & 3.16 & 3.63 & 4.12 \\
\hline 0.55 & 1.40 & 1.50 & 1.59 & 1.67 & 1.76 & 1.84 & 1.92 & 2.01 & 2.10 & 2.18 & 2.22 & 2.65 & 3.10 & 3.55 & 4.02 \\
\hline 0.60 & 1.40 & 1.49 & 1.58 & 1.66 & 1.75 & 1.83 & 1.91 & 1.99 & 2.08 & 2.15 & 2.20 & 2.62 & 3.04 & 3.47 & 3.92 \\
\hline 0.65 & 1.40 & 1.49 & 1.58 & 1.66 & 1.74 & 1.82 & 1.90 & 1.98 & 2.06 & 2.13 & 2.18 & 2.58 & 2.99 & 3.41 & 3.84 \\
\hline 0.70 & 1.40 & 1.48 & 1.57 & 1.65 & 1.73 & 1.81 & 1.89 & 1.96 & 2.04 & 2.12 & 2.16 & 2.55 & 2.95 & 3.35 & 3.77 \\
\hline 0.75 & 1.40 & 1.48 & 1.57 & 1.64 & 1.72 & 1.80 & 1.87 & 1.95 & 2.03 & 2.10 & 2.14 & 2.52 & 2.91 & 3.30 & 3.71 \\
\hline 0.80 & 1.40 & 1.48 & 1.56 & 1.64 & 1.72 & 1.79 & 1.86 & 1.94 & 2.02 & 2.09 & 2.12 & 2.50 & 2.87 & 3.25 & 3.65 \\
\hline 0.85 & 1.40 & 1.48 & 1.56 & 1.63 & 1.71 & 1.78 & 1.86 & 1.93 & 2.00 & 2.07 & 2.11 & 2.47 & 2.84 & 3.21 & 3.60 \\
\hline 0.90 & 1.40 & 1.47 & 1.55 & 1.63 & 1.70 & 1.77 & 1.85 & 1.92 & 1.99 & 2.06 & 2.10 & 2.45 & 2.81 & 3.17 & 3.55 \\
\hline 0.95 & 1.40 & 1.47 & 1.55 & 1.62 & 1.70 & 1.77 & 1.84 & 1.91 & 1.98 & 2.05 & 2.08 & 2.43 & 2.78 & 3.14 & 3.51 \\
\hline 1.00 & 1.40 & 1.47 & 1.55 & 1.62 & 1.69 & 1.76 & 1.83 & 1.90 & 1.97 & 2.04 & 2.07 & 2.41 & 2.76 & 3.11 & 3.48 \\
\hline 1.10 & 1.39 & 1.47 & 1.54 & 1.61 & 1.68 & 1.75 & 1.82 & 1.89 & 1.95 & 2.02 & 2.05 & 2.38 & 2.71 & 3.05 & 3.41 \\
\hline 1.20 & 1.39 & 1.46 & 1.54 & 1.61 & 1.68 & 1.74 & 1.81 & 1.87 & 1.94 & 2.00 & 2.03 & 2.35 & 2.67 & 3.01 & 3.36 \\
\hline 1.30 & 1.39 & 1.46 & 1.53 & 1.60 & 1.67 & 1.73 & 1.80 & 1.86 & 1.92 & 1.99 & 2.02 & 2.33 & 2.64 & 2.97 & 3.31 \\
\hline 1.40 & 1.39 & 1.46 & 1.53 & 1.60 & 1.66 & 1.72 & 1.79 & 1.85 & 1.91 & 1.97 & 2.00 & 2.30 & 2.61 & 2.94 & 3.28 \\
\hline 1.50 & 1.39 & 1.46 & 1.53 & 1.59 & 1.66 & 1.72 & 1.78 & 1.84 & 1.90 & 1.96 & 1.99 & 2.28 & 2.59 & 2.91 & 3.24 \\
\hline 1.60 & 1.39 & 1.46 & 1.52 & 1.59 & 1.65 & 1.71 & 1.77 & 1.83 & 1.89 & 1.95 & 1.98 & 2.27 & 2.57 & 2.88 & 3.21 \\
\hline 1.70 & 1.38 & 1.46 & 1.52 & 1.58 & 1.64 & 1.70 & 1.76 & 1.82 & 1.88 & 1.94 & 1.96 & 2.25 & 2.55 & 2.86 & 3.19 \\
\hline 1.80 & 1.38 & 1.45 & 1.52 & 1.58 & 1.64 & 1.70 & 1.76 & 1.81 & 1.87 & 1.93 & 1.95 & 2.24 & 2.53 & 2.84 & 3.16 \\
\hline 1.90 & 1.38 & 1.45 & 1.52 & 1.58 & 1.64 & 1.69 & 1.75 & 1.81 & 1.86 & 1.92 & 1.95 & 2.23 & 2.52 & 2.82 & 3.14 \\
\hline 2.00 & 1.38 & 1.45 & 1.51 & 1.57 & 1.63 & 1.69 & 1.74 & 1.80 & 1.86 & 1.91 & 1.94 & 2.21 & 2.50 & 2.81 & 3.13 \\
\hline 3.00 & 1.37 & 1.44 & 1.49 & 1.55 & 1.60 & 1.65 & 1.70 & 1.75 & 1.80 & 1.85 & 1.88 & 2.14 & 2.41 & 2.70 & 3.01 \\
\hline 4.00 & 1.36 & 1.43 & 1.48 & 1.53 & 1.58 & 1.63 & 1.68 & 1.73 & 1.77 & 1.82 & 1.85 & 2.10 & 2.37 & 2.65 & 2.95 \\
\hline 5.00 & 1.36 & 1.42 & 1.47 & 1.52 & 1.57 & 1.62 & 1.66 & 1.71 & 1.76 & 1.81 & 1.83 & 2.08 & 2.34 & 2.62 & 2.91 \\
\hline 6.00 & 1.36 & 1.41 & 1.47 & 1.52 & 1.56 & 1.61 & 1.65 & 1.70 & 1.75 & 1.79 & 1.81 & 2.06 & 2.32 & 2.60 & 2.89 \\
\hline 7.00 & 1.35 & 1.41 & 1.46 & 1.51 & 1.56 & 1.60 & 1.64 & 1.69 & 1.74 & 1.78 & 1.81 & 2.05 & 2.31 & 2.58 & 2.87 \\
\hline 8.00 & 1.35 & 1.41 & 1.46 & 1.51 & 1.55 & 1.59 & 1.63 & 1.68 & 1.73 & 1.78 & 1.80 & 2.04 & 2.30 & 2.57 & 2.86 \\
\hline 9.00 & 1.35 & 1.41 & 1.46 & 1.51 & 1.55 & 1.58 & 1.63 & 1.68 & 1.73 & 1.77 & 1.79 & 2.04 & 2.29 & 2.56 & 2.85 \\
\hline 10.00 & 1.35 & 1.41 & 1.46 & 1.50 & 1.54 & 1.58 & 1.63 & 1.68 & 1.72 & 1.76 & 1.79 & 2.03 & 2.29 & 2.56 & 2.84 \\
\hline 20.00 & 1.35 & 1.40 & 1.45 & 1.48 & 1.52 & 1.56 & 1.61 & 1.66 & 1.69 & 1.73 & 1.77 & 2.00 & 2.25 & 2.51 & 2.79 \\
\hline 30.00 & 1.35 & 1.40 & 1.45 & 1.47 & 1.51 & 1.56 & 1.61 & 1.66 & 1.68 & 1.72 & 1.76 & 1.98 & 2.22 & 2.48 & 2.75 \\
\hline 40.00 & 1.35 & 1.40 & 1.45 & 1.46 & 1.51 & 1.56 & 1.61 & 1.64 & 1.67 & 1.72 & 1.75 & 1.97 & 2.21 & 2.47 & 2.74 \\
\hline 50.00 & 1.35 & 1.40 & 1.45 & 1.46 & 1.50 & 1.55 & 1.61 & 1.64 & 1.67 & 1.72 & 1.74 & 1.96 & 2.20 & 2.46 & 2.73 \\
\hline 60.00 & 1.35 & 1.40 & 1.44 & 1.46 & 1.50 & 1.55 & 1.60 & 1.63 & 1.67 & 1.72 & 1.73 & 1.95 & 2.19 & 2.45 & 2.72 \\
\hline 70.00 & 1.35 & 1.40 & 1.44 & 1.45 & 1.50 & 1.55 & 1.60 & 1.62 & 1.66 & 1.72 & 1.73 & 1.95 & 2.19 & 2.45 & 2.72 \\
\hline 80.00 & 1.35 & 1.40 & 1.44 & 1.45 & 1.50 & 1.55 & 1.60 & 1.62 & 1.66 & 1.71 & 1.72 & 1.95 & 2.19 & 2.44 & 2.71 \\
\hline 90.00 & 1.35 & 1.40 & 1.44 & 1.45 & 1.50 & 1.55 & 1.60 & 1.62 & 1.66 & 1.71 & 1.72 & 1.95 & 2.18 & 2.44 & 2.71 \\
\hline 100.00 & 1.35 & 1.40 & 1.43 & 1.45 & 1.50 & 1.55 & 1.60 & 1.61 & 1.66 & 1.71 & 1.72 & 1.94 & 2.18 & 2.44 & 2.70 \\
\hline
\end{tabular}




$$
\Omega_{\text {avg }}^{(1, l) *}=\frac{\Omega_{d}^{(1, l)^{*}}+\mathrm{Kn}\left(0.9 \Omega_{d}^{(1, l)^{*}}+0.1 \Omega_{s}^{(1, l)^{*}}\right)-0.9 \mathrm{Kn}\left(\Omega_{d}^{(1, l)^{*}}-\Omega_{s}^{(1, l) *}\right) /\left[1+(R / 2.5)^{15}\right]}{1+\mathrm{Kn}} .
$$

Our recent studies (not reported here) have suggested that in addition to particle size $\varphi$ may also be influenced by temperature, the potential energy of interactions between the gas molecules and particle, and thus the particle material. We nonetheless assume here that $\varphi$ is a function of particle size only and use Eq. (39) to obtain the average collision integral $\Omega_{a v g}^{(1, l)^{*}}$, since a quantitative description of the influences of temperature and potential energy on $\varphi$ is not available.

\section{THERMOPHORETIC VELOCITY}

Following Waldmann [18], we define the thermophoretic velocity $V_{T}$ as the terminal velocity of vanishing total force on the particle, i.e., $\mathbf{F}_{T}+\mathbf{F}_{D}=\mathbf{0}$, and obtain the expression for $V_{T}$ as

$$
V_{T}=\left(1-\frac{6}{5} \frac{\Omega_{\text {avg }}^{(1,2) *}}{\Omega_{\text {avg }}^{(1,1)^{*}}}\right) \frac{\kappa \nabla T}{N k T} .
$$

For the specular and diffuse scattering cases, one needs only to replace the subscript " $a v g$ " in the collision integrals by " $s$ " and " $d$." As expected, Eq. (40) reduces to the exact $V_{T}$ equation of Waldmann [Eq. (2)] for rigid body collision if $\varphi$ is assumed to be a constant.

Based on Eq. (40), we calculate that for air at the ambient condition $V_{T} \approx 5 \mathrm{~m} / \mathrm{s}$, even if the temperature gradient is as large as $10^{3} \mathrm{~K} / \mathrm{cm}$. In comparison the mean velocity of air molecules is around $470 \mathrm{~m} / \mathrm{s}$. Therefore we expect that the

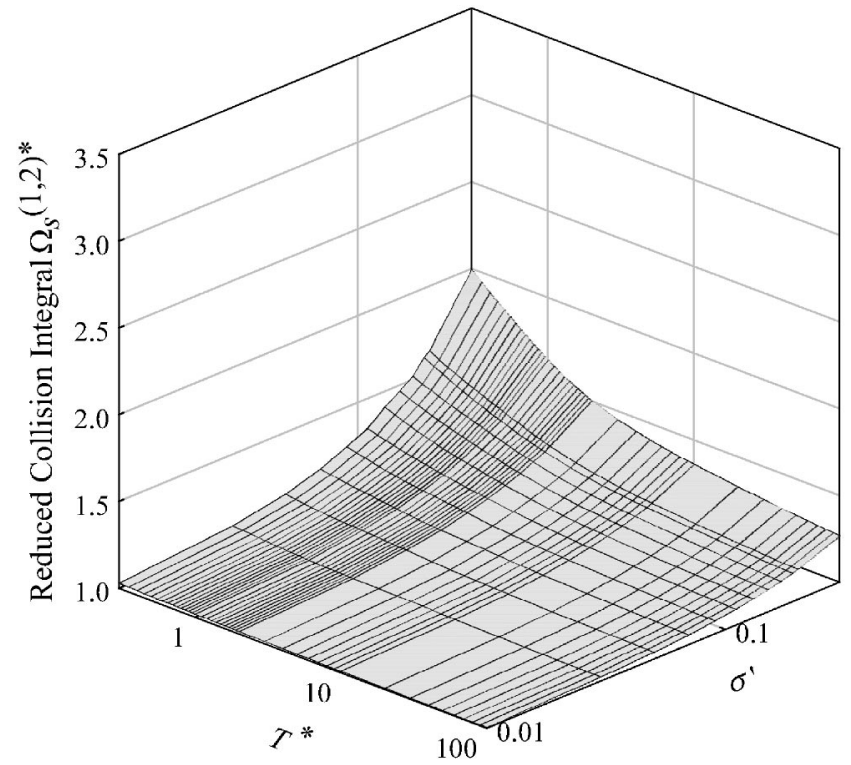

FIG. 2. Variation of the reduced collision integral $\Omega_{s}^{(1,2) *}$ as a function of modified reduced temperature $T^{*}=k T / \varepsilon^{\prime}$ and reduced collision diameter $\sigma^{\prime}$. assumption of $V \ll v$ leads to merely $1 \%$ error in $\mathbf{F}_{T}$ and $V_{T}$ (see Sec. II).

We now examine the effect of nonrigid body collisions on the thermophoretic velocity. Figure 4 shows the ratio of nonrigid body $V_{T}$ to rigid body $V_{T}$ for several types of particles. The material properties used in the calculation are found in Table IV of [2]. Soot is assumed to be composed of carbon only and its density is chosen to be $1.8 \mathrm{~g} / \mathrm{cm}^{3}$ [38]. In this figure, the solid lines are calculated using $\varphi \equiv 0.9$ for the Waldmann thermophoretic velocity, a value traditionally used in computing the thermophoretic velocity $[9,18]$ and the drag force $[19,20]$. Dashed lines in Fig. 4(c) are similarly calculated with the exception that Eq. (38) is used for $\varphi$ in Waldmann's equation.

As seen in Fig. 4, the variation of this velocity ratio is attributable both to the variation of the momentum accommodation function and, more importantly, to the potential force of interactions between the gas molecules and particle. The zigzag behavior in this ratio at $R=2-3 \mathrm{~nm}$ is largely caused by the switching of the dominant scattering mechanisms from specular to diffuse reflections, which subsequently affect the drag force. For ultrasmall particles, the deviation of Waldmann's thermophoretic velocity from the current formulation is severe, by more than an order of magnitude in some cases. For $R>10 \mathrm{~nm}$, the difference is drastically reduced compared to that for $R<10 \mathrm{~nm}$. Even then, the rigid body assumption can overpredict the thermophoretic velocity by as much as $20 \%$ for $R=10 \mathrm{~nm}$. The strong influence of potential interactions on the thermo-

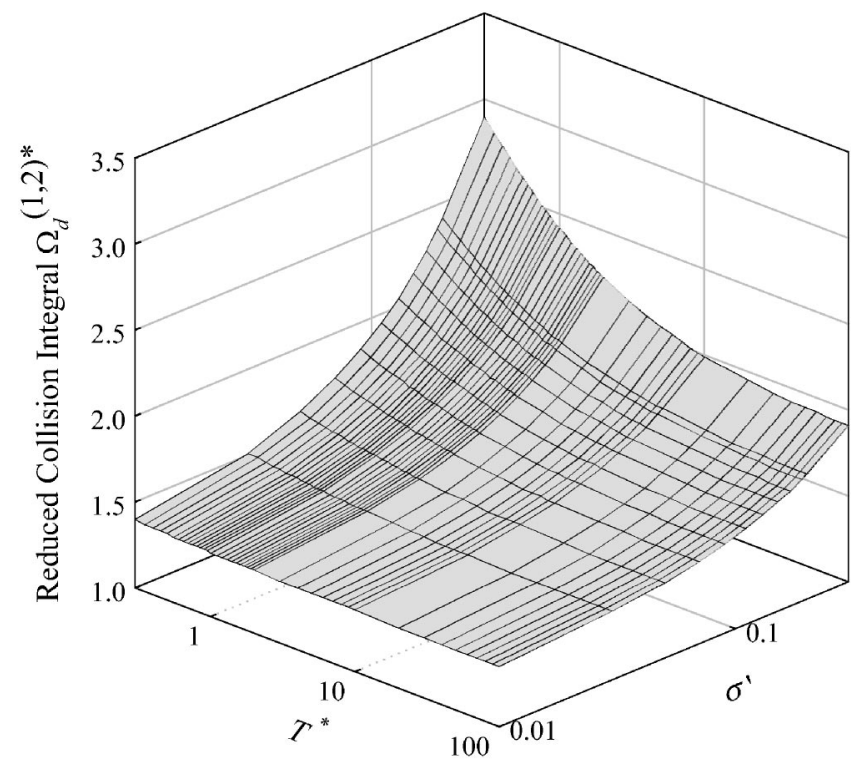

FIG. 3. Variation of the reduced collision integral $\Omega_{d}^{(1,2) *}$ as a function of modified reduced temperature $T^{*}=k T / \varepsilon^{\prime}$ and reduced collision diameter $\sigma^{\prime}$ 
TABLE III. Coefficients of Eq. (37). Coefficients for $\Omega_{s / d}^{(1,1) *}$ are obtained from [2] and those for $\Omega_{s / d}^{(1,2) *}$ are from the present study.

\begin{tabular}{lccccccc}
\hline \hline Integral & $a$ & $a_{T_{0}}$ & $a_{T_{1}}$ & $a_{T_{2}}$ & $b_{T_{0}}$ & $b_{T_{1}}$ & $b_{T_{2}}$ \\
\hline$\Omega_{s}^{(1,1)^{*}}$ & 0 & 0.316 & 1.470 & 0.476 & 1.530 & -5.013 & 4.025 \\
$\Omega_{s}^{(1,2)^{*}}$ & 0 & 0.338 & 1.315 & 0.412 & 1.503 & -4.654 & 3.410 \\
$\Omega_{d}^{(1,1)^{*}}$ & $\pi / 8$ & 1.072 & 2.078 & 1.261 & 3.285 & -8.872 & 5.225 \\
$\Omega_{d}^{(1,2) *}$ & $5 \pi / 48$ & 1.159 & 1.506 & 1.204 & 3.028 & -7.719 & 4.180 \\
\hline \hline
\end{tabular}

phoretic velocity is not surprising considering that the same effect was observed for the nanoparticle drag force $[1,2]$. By considering the potential interactions, we showed that the predictions of the theory were in excellent agreement with data obtained for silver, copper oxide, and protein nanoparticles with sizes ranging from 1 to $10 \mathrm{~nm}$ in diameter (see Figs. 6 and 8 of [2]).

In addition, we compare the thermophoretic velocity Eq. (40) with the thermal diffusion velocity predicted by the
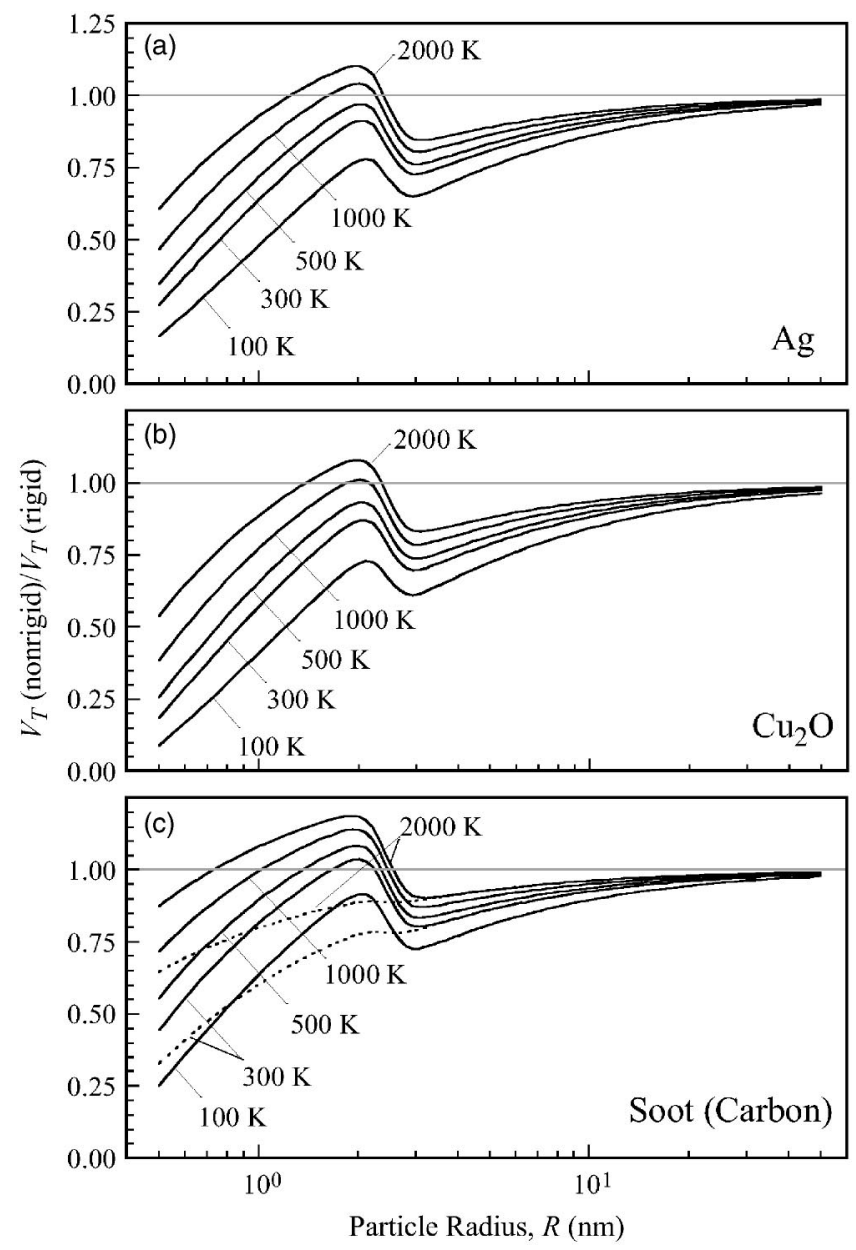

FIG. 4. Ratios of nonrigid to rigid body thermophoretic velocity calculated by dividing Eq. (40) by Waldmann's equation (2). The molecular parameters are found in Table IV of [2]. Solid lines are calculated using Eq. (38) for the momentum accommodation function and $\varphi \equiv 0.9$ for the Waldmann thermophoretic velocity. Dashed lines are similarly calculated with the exception that Eq. (38) is used for $\varphi$ in Waldmann's equation.
Chapman-Enskog theory [33]. As the particle size is reduced to molecular level, $\Omega_{\text {avg }}^{(1, l)^{*}}$ becomes $\Omega_{s}^{(1, l)^{*}}$ [2]. In a dilute particle-gas mixture the Chapman-Enskog thermophoretic velocity may be simplified to

$$
V_{T}^{C E}=C\left(1-\frac{6}{5} \frac{\Omega_{s}^{(1,2) *}}{\Omega_{s}^{(1,1)^{*}}}\right) \frac{\kappa \nabla T}{N k T}
$$

by assuming that the molar mass of the particle is much larger than that of the carrier species. In the above equation, $C$ represents a higher-order correction to $V_{T}$ and may be expressed in terms of a number of reduced collision integrals [33],

$$
C=\frac{6 \sqrt{2}\left(2 \Omega_{s}^{(2,2) *}+5 \Omega_{s}^{(1,1)^{*}}\right)}{16 \Omega_{s}^{(2,2)^{*}}-60 \Omega_{s}^{(1,2)^{*}}+48 \Omega_{s}^{(1,3)^{*}}+55 \Omega_{s}^{(1,1)^{*}} .}
$$

For rigid body collision, it is easy to find that $C \equiv 1$. For nonrigid body collision, the coefficient $C$ is listed in Table IV as a function of reduced temperatures and for both the Lennard-Jones (LJ) (12-6) potential function and the current (9-3) potentials employed for particle-gas interactions. It is seen that $C$ is close to unity for most cases. At high temperatures the correction amounts to about $10 \%$ for the LJ (12-6) potential, which is probably smaller than the uncertainty in the potential function. Therefore, as the particle size approaches that of a molecule, the current theory is quite accurate compared to the Chapman-Enskog theory.

\section{SUMMARY}

We extended our previous kinetic theory analysis of drag on particles in a low-density gas to thermophoresis of nanoparticles in the free molecule regime. Formulas for thermophoretic forces and velocities were derived on the basis of gas-kinetic theory. Our derivation considered the influence of the potential energy of interactions between the gas molecules and particle. This influence was expressed in terms of reduced collision integrals. We demonstrated that the as-

TABLE IV. Coefficients $C$ in Eq. (42) as a function of reduced temperature $T^{*}$.

\begin{tabular}{lcccc}
\hline \hline & \multicolumn{4}{c}{$T^{*}$} \\
\cline { 2 - 5 } Potential function $\Phi(r)$ & 0.3 & 1 & 10 & 20 \\
\hline Lennard-Jones (12-6) & 1.002 & 0.950 & 0.925 & 0.918 \\
Equation (36) with $\sigma^{\prime}=1$ & 1.060 & 1.034 & 1.018 & 1.017 \\
\hline \hline
\end{tabular}


sumption of a rigid body collision in Waldmann's analysis can lead to thermophoretic velocity values significantly different from the more realistic nonrigid body model, especially for nanosized particles. The current analysis also demonstrated that Waldmann's formula may be considered as a limiting case of the current result under the assumption of rigid body collisions.

On the basis of the current analysis, we propose that the thermophoretic force and velocity in the free molecule regime be modeled by

$$
\mathbf{F}_{T}=\frac{8}{3} \sqrt{\frac{2 \pi m_{r}}{k T}} R^{2} \kappa \nabla T\left(\Omega_{\text {avg }}^{(1,1) *}-\frac{6}{5} \Omega_{\text {avg }}^{(1,2) *}\right) \mathbf{u}
$$

and

$$
V_{T}=\left(1-\frac{6}{5} \frac{\Omega_{a v g}^{(1,2)^{*}}}{\Omega_{a v g}^{(1,1)^{*}}}\right) \frac{\kappa \nabla T}{N k T}
$$

respectively. In these equations the average reduced collision integrals can be evaluated by

$$
\Omega_{\text {avg }}^{(1, l) *}=\frac{\Omega_{d}^{(1, l) *}+\operatorname{Kn}\left(0.9 \Omega_{d}^{(1, l) *}+0.1 \Omega_{s}^{(1, l) *}\right)-0.9 \operatorname{Kn}\left(\Omega_{d}^{(1, l) *}-\Omega_{s}^{(1, l) *}\right) /\left[1+(R / 2.5)^{15}\right]}{1+\operatorname{Kn}},
$$

where the values of $\Omega_{s}^{(1, l) *}$ and $\Omega_{d}^{(1, l) *}$ are found in Tables I and II of [2] for $l=1$ and Tables I and II of the current paper for $l=2$. Alternatively, these collision integrals may be evaluated using the parametrized equation

$$
\Omega_{s / d}^{(1,2) *}=1+a+\left[a_{T_{0}}+\frac{a_{T_{1}}}{T^{* 1 / 4}}+\frac{a_{T_{2}}}{T^{* 1 / 2}}\right] \sigma^{\prime}+\left[b_{T_{0}}-\frac{b_{T_{1}}}{T^{* 1 / 4}}+\frac{b_{T_{2}}}{T^{*}}\right] \sigma^{\prime 2},
$$

where the coefficients $a$ and $b$ are found in Table III.

\section{ACKNOWLEDGMENTS}

This work was supported in part by the National Science Foundation (Grant No. CHE-0089136) and by the NASA Microgravity Research Program (Grant No. NCC3-843).

[1] Z. Li and H. Wang, Phys. Rev. E 68, 061206 (2003).

[2] Z. Li and H. Wang, Phys. Rev. E 68, 061207 (2003).

[3] P. S. Epstein, Phys. Rev. 23, 710 (1924).

[4] C. N. Davies, Aerosol Science (Academic, New York, 1966).

[5] J. Tyndall, Proc. Roy. Inst. 6, 3 (1870).

[6] Note that the thermophoresis phenomenon discussed herein is fundamentally different from that discussed in the context of electrokinetic phenomenon in colloids; see J. L. Anderson, Annu. Rev. Fluid Mech. 21, 61 (1989).

[7] B. H. Kaye, Direct Characterization of Fine Particles (Wiley, New York, 1981).

[8] D. Braun and A. Libchaber, Phys. Rev. Lett. 89, 188103 (2002).

[9] A. Gomez and D. E. Rosner, Combust. Sci. Technol. 89, 335 (1993).

[10] B. Zhao, K. Uchikawa, J. C. McCormick, C. Y. Ni, J. G. Chen, and H. Wang, Proc. Combust. Inst.

[11] P. S. Epstein, Z. Phys. 54, 537 (1929).

[12] J. R. Brock, J. Colloid Sci. 17, 768 (1962).

[13] H. A. Dwyer, Phys. Fluids 10, 977 (1967).

[14] Y. Sone and K. Aoki, in Rarefied Gas Dynamics, edited by J. L. Potter (AIAA, New York, 1977), p. 417.

[15] Y. Sone and K. Aoki, in Rarefied Gas Dynamics, edited by S. Fisher (AIAA, New York, 1981), p. 489.

[16] S. R. de Groot and P. Mazur, Non-equilibrium Thermodynamics (North-Holland, Amsterdam, 1969).
[17] M. E. Schimpf and S. N. Semenov, J. Phys. Chem. B 104, 9935 (2000).

[18] L. Waldmann, Z. Naturforsch. A 14a, 589 (1959).

[19] M. D. Allen and O. G. Raabe, J. Aerosol Sci. 13, 537 (1982).

[20] R. A. Millikan, Phys. Rev. 22, 1 (1923).

[21] E. A. Mason and S. Chapman, J. Chem. Phys. 36, 627 (1962).

[22] L. Monchick, K. S. Yun, and E. A. Mason, J. Chem. Phys. 39, 654 (1963).

[23] W. Li and E. J. Davis, J. Aerosol Sci. 26, 1063 (1995).

[24] W. Li and E. J. Davis, J. Aerosol Sci. 26, 1085 (1995).

[25] N. T. Tong, J. Colloid Interface Sci. 51, 143 (1975).

[26] F. Prodi, G. Santachiara, and V. Prodi, J. Aerosol Sci. 10, 421 (1979).

[27] S. K. Loyalka, J. Aerosol Sci. 23, 291 (1992).

[28] S. Takata, K. Aoki, and Y. Sone, in Rarefied Gas Dynamics, edited by B. D. Shizgal and D. P. Weaver (AIAA, New York, 1994), p. 626.

[29] J. R. Brock, J. Colloid Interface Sci. 23, 448 (1967); 25, 392 (1967).

[30] L. Talbot, R. K. Cheng, R. W. Schefer, and D. R. Willis, J. Fluid Mech. 101, 737 (1980).

[31] K. Yamamato and Y. Ishihara, Phys. Fluids 31, 3618 (1988).

[32] F. Zheng, Adv. Colloid Interface Sci. 97, 255 (2002).

[33] S. Chapman and T. G. Cowling, The Mathematical Theory of Non-Uniform Gases (Cambridge University Press, Cambridge, England, 1970). 
[34] G. A. Bird, Molecular Gas Dynamics (Oxford University Press, London, 1976).

[35] R. A. Millikan, Philos. Mag. 34, 1 (1917).

[36] J. O. Hirschfelder, C. F. Curtiss, and R. B. Bird, Molecular Theory of Gases and Liquids (Wiley, New York, 1954).
[37] V. Ya. Rudyak and S. L. Krasnolutski, Dokl. Phys. 46, 897 (2001).

[38] S. J. Harris and A. M. Weiner, Combust. Sci. Technol. 31, 155 (1983). 